\title{
A modified Delphi study of screening for fetal alcohol spectrum disorders in Australia
}

\author{
Rochelle E Watkins ${ }^{1 *}$, Elizabeth J Elliott ${ }^{2,3,4}$, Jane Halliday ${ }^{5,6}$, Colleen M O'Leary ${ }^{1,7}$, Heather D'Antoine ${ }^{8}$, \\ Elizabeth Russell ${ }^{9}$, Lorian Hayes ${ }^{10}$, Elizabeth Peadon ${ }^{2,3}$, Amanda Wilkins $^{1,11}$, Heather M Jones ${ }^{1}$, Anne McKenzie ${ }^{1}$, \\ Sue Miers ${ }^{12}$, Lucinda Burns ${ }^{13}$, Raewyn C Mutch ${ }^{1,11}$, Janet M Payne ${ }^{1}$, James P Fitzpatrick ${ }^{2,4}$, Maureen Carter ${ }^{14}$, \\ Jane Latimer ${ }^{4}$ and Carol Bower ${ }^{1}$
}

\begin{abstract}
Background: There is little reliable information on the prevalence of fetal alcohol spectrum disorders (FASD) in Australia and no coordinated national approach to facilitate case detection. The aim of this study was to identify health professionals' perceptions about screening for FASD in Australia.

Method: A modified Delphi process was used to assess perceptions of the need for, and the process of, screening for FASD in Australia. We recruited a panel of 130 Australian health professionals with experience or expertise in FASD screening or diagnosis. A systematic review of the literature was used to develop Likert statements on screening coverage, components and assessment methods which were administered using an online survey over two survey rounds.

Results: Of the panel members surveyed, 95 (73\%) responded to the questions on screening in the first survey round and, of these, 81 (85\%) responded to the second round. Following two rounds there was consensus agreement on the need for targeted screening at birth (76\%) and in childhood (84\%). Participants did not reach consensus agreement on the need for universal screening at birth (55\%) or in childhood (40\%). Support for targeted screening was linked to perceived constraints on service provision and the need to examine the performance, costs and benefits of screening.

For targeted screening of high risk groups, we found highest agreement for siblings of known cases of FASD (96\%) and children of mothers attending alcohol treatment services (93\%). Participants agreed that screening for FASD primarily requires assessment of prenatal alcohol exposure at birth (86\%) and in childhood (88\%), and that a checklist is needed to identify the components of screening and criteria for referral at birth (84\%) and in childhood (90\%).

Conclusions: There is an agreed need for targeted but not universal screening for FASD in Australia, and sufficient consensus among health professionals to warrant development and evaluation of standardised methods for targeted screening and referral in the Australian context. Participants emphasised the need for locally-appropriate, evidence-based approaches to facilitate case detection, and the importance of ensuring that screening and referral programs are supported by adequate diagnostic and management capacity.
\end{abstract}

\section{Background}

Prenatal alcohol exposure causes a range of disorders of fetal development [1-3]. The term fetal alcohol spectrum disorders (FASD) is used to identify one group of conditions that can result from prenatal exposure to alcohol [4]. Fetal alcohol spectrum disorders feature characteristic combinations of facial anomalies, growth impairment,

\footnotetext{
* Correspondence: rwatkins@ichr.uwa.edu.au

${ }^{1}$ Telethon Institute for Child Health Research, Centre for Child Health

Research, The University of Western Australia, Perth, Australia

Full list of author information is available at the end of the article
}

intellectual disability, behavioural disorders and birth defects [5-8], although the spectrum of disorders most commonly includes impairments of neurological function that are not accompanied by characteristic facial anomalies or growth deficit $[9,10]$. There is some variation in the range of disorders recognised within the spectrum and the use of specific diagnostic terminology between different diagnostic guidelines. However, all guidelines include the well-established diagnostic category of fetal alcohol syndrome (FAS) $[6,8,11-13]$, and most include diagnostic categories for

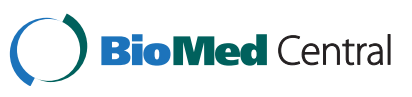


partial FAS (PFAS), and alcohol-related neurodevelopmental disorder (ARND) or its equivalent $[6,8,11,13]$.

The diagnosis of FASD is often delayed or missed [4,14-16], despite evidence linking diagnosis and intervention to improved educational, social and health outcomes $[17,18]$. Failure to identify children with FASD has been attributed to the limited ability of health service providers to access affected children and their mothers, a lack of accurate routine screening for FASD or of routine screening for maternal alcohol use during pregnancy, limited clinical capacity to recognise and diagnose these disorders, and the difficulty of identifying all but the most severe cases at birth $[19,20]$. The identification of FASD in Australia is likely to be limited by poor awareness of diagnostic features among paediatricians [21] and other health professionals [22], and the absence of national guidelines for diagnosis. Concern about stigma as a result of diagnosis [22] may also influence the willingness to diagnose or to be diagnosed.

There is only limited information available on the prevalence of FASD in Australia [15], with no routine screening or surveillance established for the disorders. The need to address significant gaps in the capacity to identify and diagnose FASD in Australia is reflected in recent health policy [23] and the launch of an Australian federal government inquiry into the incidence and prevention of FASD in 2011 [24]. Although not yet implemented, the Western Australian FASD Model of Care [23] recommends multi-stage population screening to identify at risk newborns and children based on history of maternal alcohol consumption, abnormal growth parameters and developmental delay. Targeted screening is also recommended in a number of sub-groups considered at high risk, including children of mothers who receive alcohol treatment services and children in state care.

Screening for FASD provides a means to facilitate referral, diagnosis and support [6]; however, several factors complicate the identification of, and screening for, FASD [12]. Screening for FAS has primarily utilised identification of the three characteristic FAS facial anomalies (short palpebral fissures, smooth philtrum and thin upper lip) that are collectively specific to FAS [25]; although routinely collected data from neonatal medical records including growth, head circumference, maternal alcohol use and other criteria have also been used [26]. Screening for FAS using facial photographs to detect the characteristic facial anomalies has been found to be effective in high-risk populations in North America, with a positive predictive value of $86 \%$ and a negative predictive value of $100 \%$ [27].

Screening for FASD is more difficult because facial dysmorphology is frequently absent, impairments of neurological function are predominant [6], and the neurobehavioural features are not unique to FASD [28].
Inconsistency in the methods used for screening and referral internationally [29] is linked to a lack of screening instruments for FASD that are specific and sensitive to the fetal effects of prenatal alcohol exposure [6]. Biochemical screening has the potential to facilitate early diagnosis; however, apart from the detection of ethyl esters in newborn meconium, screening methods are not well established [30,31]. Heterogeneity among the observed features of FASD due to variation in genetic factors, patterns of alcohol exposure and other maternal risk factors adds to the complexity of screening for FASD [29]. Nevertheless, the use of standard screening processes may facilitate the appropriate identification of individuals who require specialist diagnostic assessment.

Screening programs for both FAS and FASD frequently use assessment methods that are directly linked to diagnostic criteria. Published screening assessments have included prenatal alcohol exposure, weight, height, head circumference, developmental delay, learning difficulties, behavioural problems and the presence of characteristic FAS facial anomalies [9,26,32]. However, screening programs do not always assess the full range of diagnostic features. For example, facial dysmorphology assessment has been excluded from FASD screening criteria $[9,32]$, consistent with the recommended need for experienced assessors, the infrequent occurrence of facial anomalies among individuals with FASD, and the lack of norms for ethnically diverse populations [29].

There is little locally-relevant evidence on which to base policy decisions about FASD screening in Australia. There is no coordinated national approach to facilitate the identification of FASD in Australia, and it is unclear to what extent current policy and capacity for service delivery are consistent with clinician perspectives of priorities and needs. We aimed to evaluate perceptions about screening for FASD among Australian health professionals with experience or expertise in FASD screening or diagnosis to guide research and policy on FASD screening in Australia.

\section{Methods}

A modified Delphi process [33] was used to assess Australian health professionals' perceptions of the need for and process of FASD screening in Australia. The modified Delphi design generally diverges from the classical Delphi method in the use of alternative means to derive the content of the initial quantitative questionnaire round [33] while still allowing the collection of rich data based on multiple questionnaire iterations [34]. Modified Delphi studies are particularly appropriate where relevant knowledge exists.

\section{Questionnaire development}

An existing systematic review on FASD screening and diagnosis [35] was used to identify literature relevant to 
the design of screening for FASD in Australia. The search strategy for this review was updated to include literature published up to and including September 2010, and expanded to include additional search terms (fetal alcohol effects, fetal alcohol disorders, partial fetal alcohol syndrome, alcohol related birth defects, alcohol related neurodevelopmental disorder, screen\$, diagnos\$). Additional relevant databases were also searched (CINAHL, PsychINFO and Web of Science).

Descriptions of screening programs identified in the published literature were used to design Likert statements to evaluate perceptions about screening coverage, screening components, and assessment methods. Participants were asked to rate their agreement with each statement on a 5-point Likert scale which ranged from 'strongly agree' to 'strongly disagree', and a response option of 'no comment' was provided to enable participants to indicate that a statement was outside their area of expertise. Each question area also included open ended questions and participants were encouraged to provide comments on their responses and identify where the statements did not reflect their beliefs. The questions on screening were administered as part of a larger survey on the screening and diagnosis of FASD in Australia. Perceptions about the diagnosis of FASD have been reported elsewhere [36]. The questionnaire was pilot tested with 16 health professionals and health researchers to examine coherence, feasibility and face validity prior to administration.

\section{Panel recruitment}

We aimed to recruit a large panel of Australian health professionals with experience or expertise in FASD screening or diagnosis. Panel members were identified using three purposive sampling strategies [37]: i) medical practitioners who reported a diagnosis of FAS to the Australian Paediatric Surveillance Unit (APSU) in a previous study [15], ii) health professionals who were identified by study investigators as having experience or expertise in FASD screening or diagnosis, and iii) individuals who responded to calls to health professional organisations for individuals who had relevant experience or expertise.

Recruitment of panel members with relevant experience or expertise underpins the validity of this study, and processes used to enrol panel members varied by sampling strategy based on the need to confirm relevant expertise prior to enrolment. Information about this study was distributed to 57 eligible medical practitioners who had reported a diagnosis of FAS to the APSU, and all were included in the study apart from 17 who either actively declined to participate or who could not be contacted (based on an invalid email address or an automated email reply). In contrast, all other invited health professionals were only included in the panel if they actively confirmed their suitability and willingness to participate. Among these 149 invited health professionals, 90 accepted the invitation to participate. The modified Delphi process commenced with 130 Australian panel members: including 40 who had reported a diagnosis of FAS to the APSU, 59 who were identified as having experience or expertise in FASD screening or diagnosis, and 31 who were recruited from professional organisations.

\section{Questionnaire administration}

The online password-protected questionnaire was administered from a secure web server, and responses were automatically saved to a secure MySQL database. All panel members were invited by email to complete the first round questionnaire within 14 days, and two email reminders were sent prior to the round deadline. Due to requests for a longer period for response, the round 1 deadline was extended by 8 days, and 21 days was provided for response to the round 2 questionnaire. Where contact details were available, panel members who had not visited the study website were targeted for additional follow-up. One contact attempt was made by telephone at least 5 days before the closure date of rounds 1 and 2 . Participants who did not complete round 1 were excluded from round 2.

\section{Round 2 questionnaire revision}

Both quantitative and qualitative responses were considered in the questionnaire revision process. Due to the length of the round 1 questionnaire, revisions aimed to minimise the length of the round 2 questionnaire and exclude question areas that had achieved consensus. If at least $70 \%$ of participants agreed or strongly agreed with a statement, it was considered endorsed. This level of consensus was decided a priori. If fewer than $60 \%$ of participants agreed or strongly agreed with a statement, the statement was rejected or modified based on qualitative findings. However, where multiple statements were related to the same issue, consensus was sought for the question area rather than for each statement in isolation. As such, several statements that either achieved consensus agreement or less than $60 \%$ agreement were included in round 2 where clear consensus was not achieved for that question area. New statements were also included in round 2 if qualitative findings indicated that the round 1 questionnaire failed to represent a relevant alternative perspective. The round 2 questionnaire included feedback of representative comments as well as group and individual per cent agreement results from round 1 to enable participants to reflect on their previous responses.

\section{Analysis}

Descriptive statistics were generated for each statement, including response frequencies and dispersion (inter- 
quartile deviation). Associations between ratings for key statements on screening coverage and individual characteristics, were explored using the Chi square test or Fisher's exact test [38]. The Wilcoxon signed rank test was used to compare agreement between statements on screening coverage, and between alternative screening methods for growth. All analyses were evaluated using two-tailed test statistics.

Qualitative data were coded and analysed independently by two investigators using an inductive content analysis approach $[39,40]$. Data from each open ended question were reviewed alongside the quantitative data, compared and coded based on the underlying meaning of the responses. Both analysts' coding schemes were reviewed for consistency to ensure credibility and trustworthiness of the analysis process [39]. This study was approved by the University of Western Australia Human Research Ethics Committee and the Western Australian Aboriginal Health Information and Ethics Committee.

\section{Results}

Of the 130 panel members, 95 (73\%) responded in round 1 , and 81 of these 95 (85\%) responded in round 2. Response in round 1 was: $68 \%(27 / 40)$ among panel members who reported a diagnosis of FAS to the APSU; $78 \%$ (46/59) among panel members recruited by the study investigators; and $71 \%(22 / 31)$ among panel members recruited through professional organisations. Round 1 participants were most commonly paediatricians (46\%), and approximately three quarters reported experience in FASD screening or diagnosis (Table 1).

\section{Screening coverage}

Due to a lack of consensus on the use of targeted screening at birth following round 1, all four statements evaluating screening coverage were re-administered in round 2 (Table 2, statements 1-4). After round 2 there was no strong evidence of a difference in agreement that screening for FASD at birth should be targeted (76\%) or universal (55\%; Wilcoxon $\mathrm{Z}=-1.7, \mathrm{p}=0.09$ ). Participants most commonly agreed that screening for FASD in childhood should be targeted (84\%), and were more likely to agree that it should be targeted than universal (40\%; Wilcoxon $\mathrm{Z}=-4.1, \mathrm{p}<0.001$ ). They were also more likely to agree with the use of targeted screening in childhood compared with targeted screening at birth (Wilcoxon $\mathrm{Z}=-2.2, \mathrm{p}=0.03$ ). We found no evidence of differences in agreement about screening coverage at birth or in childhood according to experience in screening or diagnosis (all $\mathrm{p}>0.7$ ).

There was clear consensus agreement on all indications for targeted screening evaluated in round 1 (Table 2, statements 5-22). Over 95\% of participants agreed with screening for FASD where there is parental concern that
Table 1 Summary of participant characteristics in round 1

\begin{tabular}{|c|c|}
\hline Characteristic & $\begin{array}{c}n(\%) \\
(n=95)\end{array}$ \\
\hline \multicolumn{2}{|l|}{ Sex } \\
\hline Male & $24(25)$ \\
\hline Female & $71(75)$ \\
\hline \multicolumn{2}{|l|}{ State $^{\dagger}$} \\
\hline Australian Capital Territory & $1(1)$ \\
\hline New South Wales & $23(26)$ \\
\hline Northern Territory & $5(6)$ \\
\hline Queensland & $23(26)$ \\
\hline South Australia & $6(7)$ \\
\hline Tasmania & $3(3)$ \\
\hline Victoria & $4(5)$ \\
\hline Western Australia & $24(27)$ \\
\hline \multicolumn{2}{|l|}{ Occupation } \\
\hline Paediatricians & $44(46)$ \\
\hline Non-paediatrician medical practitioners & $22(23)$ \\
\hline Other health professionals ${ }^{\Delta}$ & $29(31)$ \\
\hline \multicolumn{2}{|l|}{ Experience in FASD diagnosis ${ }^{\ddagger}$} \\
\hline Yes & $38(41)$ \\
\hline No & $55(59)$ \\
\hline \multicolumn{2}{|c|}{$\begin{array}{l}\text { Experience in FASD screening, diagnosis or contributing }{ }^{*} \text { to } \\
\text { diagnosis }^{*}\end{array}$} \\
\hline Yes & $70(75)$ \\
\hline No & $23(25)$ \\
\hline \multicolumn{2}{|l|}{ Completed specific training on FASD diagnosis ${ }^{\ddagger}$} \\
\hline Yes & $20(22)$ \\
\hline No & $73(79)$ \\
\hline \multicolumn{2}{|l|}{ Work location ${ }^{\ddagger}$} \\
\hline Includes rural or remote practice & $41(44)$ \\
\hline Does not include rural or remote practice & $52(56)$ \\
\hline \multicolumn{2}{|c|}{$\begin{array}{l}{ }^{\dagger} \text { Valid } n=89 . \\
{ }^{\ddagger} \text { Valid } n=93 . \\
{ }^{\Delta} \text { Other health professionals includes nurses, allied health professionals, health } \\
\text { workers and health researchers. } \\
{ }^{*} \text { Contribution to diagnosis includes the conduct of relevant assessments that } \\
\text { inform diagnosis, but not determination of the final diagnosis. }\end{array}$} \\
\hline
\end{tabular}

their child may have a FASD; evidence of characteristic FAS facial anomalies; an alcohol-related illness or dependency in the birth mother; and a sibling with FASD.

In round 1, 61 participants (64\%) provided comments about their preferences for screening coverage. Comments most frequently indicated support for universal screening as an ideal and ethical approach that decreases the risk of missed cases, helps overcome the limitations of a potentially unreliable maternal alcohol history, and enables early diagnosis and intervention. However, targeted screening 
Table 2 Agreement with statements on screening coverage and indications in rounds 1 and 2

\begin{tabular}{|c|c|c|c|}
\hline Statement & R1 $\mathrm{N}$ & R1 \% Agree $^{\dagger}$ (IQD) & R2 \% Agree $^{\dagger}$ (IQD) \\
\hline \multicolumn{4}{|l|}{ Screening coverage } \\
\hline 1. Screening for FASD at birth should be universal & 87 & $58(3)$ & $55(3)$ \\
\hline 2. Screening for FASD at birth should be targeted & 88 & $68(2)$ & $76(1)$ \\
\hline 3. Screening for FASD in childhood should be universal & 86 & $49(3)$ & $40(2)$ \\
\hline 4. Screening for FASD in childhood should be targeted & 86 & $78(1)$ & $84(1)$ \\
\hline \multicolumn{4}{|l|}{ Indications for targeted screening - presentations } \\
\hline 5. an alcohol-related event, illness or dependency in the birth mother & 91 & $96(1)$ & - \\
\hline 6. a parent/foster parent who is concerned that their child might have a FASD & 91 & $99(1)$ & - \\
\hline 7. prenatal alcohol exposure & 90 & $92(1)$ & - \\
\hline 8. developmental delay & 88 & $91(1)$ & - \\
\hline 9. growth retardation or failure to thrive & 87 & $91(1)$ & - \\
\hline 10. structural central nervous system abnormalities & 82 & $87(1)$ & - \\
\hline 11. neurological signs & 84 & $82(1)$ & - \\
\hline 12. functional central nervous system abnormalities & 84 & $88(1)$ & - \\
\hline 13. characteristic FAS facial anomalies & 89 & $97(1)$ & - \\
\hline 14. birth defects & 85 & $93(1)$ & - \\
\hline 15. reported or observed problems with behaviour & 88 & $86(1)$ & - \\
\hline \multicolumn{4}{|l|}{ Indications for targeted screening - high risk groups } \\
\hline 16. children of mothers attending alcohol treatment services & 91 & $93(1)$ & - \\
\hline 17. siblings of identified cases of FASD & 90 & $96(1)$ & - \\
\hline 18. children who are diagnosed with ADHD & 82 & $74(2)$ & - \\
\hline 19. children entering a child development service & 89 & $87(1)$ & - \\
\hline 20. children entering child protection & 86 & $85(1)$ & - \\
\hline 21. children entering foster care or adoptive placements (incl. kinship care) & 86 & $87(1)$ & - \\
\hline 22. children entering a juvenile justice setting & 84 & $82(1)$ & - \\
\hline
\end{tabular}

R1-Round 1; R2-Round 2; IQD-inter-quartile deviation.

'Includes responses 'agree' and 'strongly agree.'

Results for statements that reached $70 \%$ agreement (consensus) are presented in bold.

of high risk groups was commonly supported as a more feasible and effective approach. Participants commonly identified practical constraints associated with screening and diagnosis, including the need for increased service capacity and provider training, the need for adequate early intervention services, and the evolving and variable nature of FASD presentations. Respondents also expressed concerns about the absence of well-established or accurate screening tests and the sensitivity, specificity and cost-effectiveness of screening. They also indicated support for the universal collection of a maternal history of alcohol use during the prenatal period and at birth to enable preventive interventions and targeted follow-up. Participants less commonly described adverse psychosocial consequences of screening or of assigning an aetiological diagnostic label such as maternal blame or stigma; and a lack of support for screening programs which focus on a single condition.

\section{Components of screening}

Consensus was achieved for all components of screening assessed apart from the testing of fatty acid esters (Table 3, statements 1-8 and 12-21). Some participants commented in round 1 that the agreed components of screening for FASD were already routinely assessed at birth or during the assessment of neurodevelopmental problems presenting in childhood; that the primary need is for health professionals to assess prenatal alcohol exposure and consider it as a potential cause of identified abnormalities; and that a screening checklist and criteria for referral are required. Additional statements were included in the round 2 questionnaire to evaluate participants' perceptions of these issues (Table 3, statements 9-11 and 22-24). Following round 2 there was no consensus agreement that information required for screening is currently routinely collected. However, there was consensus agreement that screening primarily requires 
Table 3 Agreement with statements on the components of screening at birth and in childhood in rounds 1 and 2

\begin{tabular}{|c|c|c|c|}
\hline Statement & R1 N & R1 \% Agree $^{\dagger}$ (IQD) & R2 \% Agree $^{\dagger}$ (IQD) \\
\hline \multicolumn{4}{|l|}{ Screening at birth } \\
\hline 1. prenatal alcohol exposure & 92 & $98(1)$ & - \\
\hline 2. birth weight, length and head circumference & 90 & $100(1)$ & - \\
\hline 3. fatty acid esters (FAEE) in meconium collected within 72 hours of birth & 37 & $46(3)$ & - \\
\hline 4. characteristic FAS facial anomalies & 89 & $98(1)$ & - \\
\hline 5. birth defects & 89 & $98(1)$ & - \\
\hline 6. evidence of withdrawal from alcohol or other drugs & 90 & $99(1)$ & - \\
\hline 7. family history of FASD or developmental delay & 79 & - & $95(1)$ \\
\hline $\begin{array}{l}\text { 8. evidence of CNS dysfunction including irritability, feeding difficulties or other } \\
\text { neurological signs }\end{array}$ & 77 & & 91 (1) \\
\hline $\begin{array}{l}\text { 9. most of the information required for FASD screening at birth is routinely } \\
\text { collected at birth }\end{array}$ & 72 & - & $56(2)$ \\
\hline $\begin{array}{l}\text { 10. screening for FASD at birth primarily requires health professionals to assess } \\
\text { prenatal alcohol exposure and } \\
\text { consider it as a potential cause of other relevant abnormalities identified }\end{array}$ & 79 & - & $86(1)$ \\
\hline 11. a checklist is needed to support the implementation of screening for FASD & 79 & - & $84(1)$ \\
\hline
\end{tabular}
components to be assessed and criteria for conducting a full diagnostic evaluation

\section{Screening in childhood}

12. prenatal alcohol exposure $\quad 90$

13. growth (height and weight)

14. head circumference

15. developmental delay

16. neurological signs

17. functional CNS abnormalities (e.g. cognition, behaviour disorders)

18.hearing and vision

19. characteristic FAS facial anomalies

20. birth defects

21. family history of FASD, developmental delay, abuse or neglect

22. most of the information required for FASD screening in childhood is routinely assessed as part of a

general clinical assessment of children with neurodevelopmental or other related presentations

23. screening for FASD in childhood primarily requires health professionals to assess prenatal alcohol exposure

and consider it as a potential cause of other relevant abnormalities identified

(e.g. abnormalities of

development, learning, behaviour)

24. a checklist is needed to support the implementation of screening for FASD in childhood that identifies the

components to be assessed and criteria for conducting a full diagnostic evaluation

R1-Round 1; R2-Round 2; IQD-inter-quartile deviation; CNS-central nervous system. 'Includes responses 'agree' and 'strongly agree.'

Results for statements that reached $70 \%$ agreement (consensus) are presented in bold. health professionals to assess prenatal alcohol exposure and consider it as a potential cause of other relevant abnormalities identified at birth (86\%) and in childhood $(88 \%)$; and that a screening checklist is required to identify criteria for referral at birth (84\%) and in childhood $(90 \%)$.
Participant comments linked improved awareness of and ability to screen for FASD with improved case identification. Screening for prenatal alcohol exposure, although potentially unreliable, was considered by some participants to be the most important element of screening for FASD due to the difficulty of screening for 
the spectrum of disorders at birth, and the fact that central nervous system (CNS) dysfunction is often not apparent in very young children. Other participants referred to difficulties in screening associated with nondisclosure of alcohol use during pregnancy, or where no maternal history is available. Several participants also identified the limited usefulness of screening for facial anomalies when screening for FASD.

\section{Assessment methods}

Almost all statements on screening assessment methods achieved consensus in round 1 (Table 4). There was a high level of agreement that the assessment of prenatal alcohol exposure should identify and record the number of standard drinks consumed on a typical drinking occasion, the frequency of drinking, the frequency of excessive drinking and the timing of alcohol intake during pregnancy (Table 4, statements 1-5). Most participants (71\%) believed that prenatal alcohol exposure should be assessed using a formal tool (Table 4, statements 6-7).

The most commonly recommended formal assessment tool was the AUDIT-C (21\%) [41], followed by Lifescripts (17\%) [42] which incorporates the AUDIT-C, T-ACE (14\%) and TWEAK (13\%) [43]. Most participants reported that they were not familiar with the Lifescripts (63\%), TWEAK (62\%), T-ACE (62\%) and AUDIT-C (59\%) tools, and no alternative formal assessment tools were proposed. Following round 2, the role of informal inquiry in prenatal alcohol exposure assessment was clarified, with consensus agreement for the combination of informal and formal methods of inquiry. After receiving further information about the AUDIT-C in round 2, 89\% of participants considered that it provided a useful screening tool for prenatal alcohol exposure (Table 4, statements 8-10).

Participants most frequently supported the comparison of height and weight with population standards to assess growth deficit (Table 4, statements 11-14), and there was a high level of consensus on the specific facial anomalies that should be assessed during screening (Table 4, statement 15). Participant comments in round 1 indicated a lack of distinction between screening and diagnostic assessments, the need for training to use facial anomaly assessment tools reliably, the lack of suitable Australian reference data for comparison, and the need for screening methods to be efficient. Following round 2 there was consensus agreement that formal assessment of facial anomalies could be used, but was not required, to assess facial anomalies at the screening stage (Table 4, statements 17-22).

There was agreement on the assessment of a broad range of CNS abnormalities in screening, and limited support for the use of brain imaging (Table 5, statements 1-14). Participant comments in round 1 highlighted the need to distinguish between screening and diagnostic assessments, and the need for a pragmatic approach to assessment considering cost and resources, including the possibility of qualitative assessment of core areas at the screening stage. Round 2 results indicated agreement that clinical identification or third party report were acceptable indicators of CNS abnormality at the screening stage (Table 5, statements 18-21).

\section{Response completeness}

Over half of the 95 participants (57\%) completed 10 or fewer of the round 1 Likert statements on screening. Non-complete responses most commonly included a response of 'no comment', indicating that the statements were outside participant's areas of expertise. Completion of 10 or fewer statements was more frequent among participants who reported no experience in diagnosis or screening $(87 \%)$ and health professionals other than paediatricians $(73 \%)$ than among participants who reported experience in diagnosis or screening (49\%, $\left.X^{2}=11.7, \quad \mathrm{p}=0.001\right)$ and paediatricians $\left(39 \%, \quad X^{2}=11.3\right.$, $\mathrm{p}=0.001$ ) respectively.

Assessment of the association between response completeness and perceptions found clear evidence of an association with response completeness for only one of the statements about screening coverage, components, and assessment methods. Participants who completed 10 or fewer statements were less likely to agree with the use of clinician preference and experience in the choice of neuro-behavioural assessment methods (42\%) compared with participants who completed more than 10 statements (73\%, $\chi^{2}=7.2, \mathrm{p}=0.007$ : Table 5, item 16).

\section{Discussion}

This is the first systematic evaluation of health professionals' perceptions of the need for and design of screening for FASD in Australia. We found consensus support for targeted screening, and more frequent agreement with screening in childhood than at birth. Although about half the participants supported the need for universal screening for FASD as an ideal and ethically responsible approach to minimise missed cases, consensus agreement was only achieved for targeted screening of individuals with relevant clinical presentations and groups at high risk of FASD. Parent or caregiver concern that their child may have a FASD and clinical presentations associated with the likelihood of prenatal alcohol exposure were most strongly endorsed as indications for targeted screening.

Targeted screening was considered more cost effective and feasible than universal screening, with support for targeted screening linked to constraints on service provision, including the need for provider training and increased demand for diagnostic and intervention services. Participants were also concerned about the limitations of existing screening methods and the need for programs 
Table 4 Agreement with statements on screening assessment methods for prenatal alcohol exposure, growth deficit and characteristic fetal alcohol syndrome facial anomalies in rounds 1 and 2

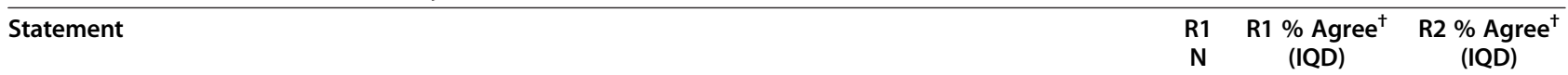

Prenatal alcohol exposure

Assessment of prenatal alcohol exposure should identify and record the:

1.... number of standard drinks consumed during a typical drinking occasion 85

2.... frequency of drinking occasions

3.... frequency of excessive (binge) drinking ( $5+$ standard drinks per occasion)

4. ... timing of alcohol intake during pregnancy

5. Alcohol exposure should be assessed alongside other lifestyle factors (e.g. diet)

6. Prenatal alcohol exposure can be effectively assessed using an informal approach (e.g. inquiring during a consultation)

7. Prenatal alcohol exposure should be assessed using a formal tool

8. The use of formal tools for the assessment of prenatal alcohol exposure should be combined with a clinical interview to obtain more detailed information about alcohol consumption patterns, potential indicators of addiction and other relevant contextual information

9. Information on alcohol use from family members, other health professionals or community members (if appropriate) should be sought if indicated

10. The AUDIT-C would be a useful tool for the formal assessment of prenatal alcohol exposure

$71(2)$

\section{Growth deficit}

11. Growth should be assessed by comparing height and weight with population standards

12. Growth should be assessed by comparing weight to height ratio with population standards

13. Growth should be assessed by comparing weights over time (to identify decelerating weight over time)

14. Assessment of growth deficit should consider other factors that may affect growth (e.g. gestational age parental size, gestational diabetes, nutritional status, illness)

\section{Characteristic fetal alcohol syndrome (FAS) facial anomalies}

15. The presence of the following characteristic FAS facial anomalies should be assessed: smooth philtrum, thin upper lip, and small palpebral fissures

16. Assessment of characteristic FAS facial anomalies should use appropriate anthropometric population standards for race and age where available

\section{At the screening stage, characteristic FAS facial anomalies can be effectively assessed using:}

17. ... clinical observation(R1) /Facial anomalies can be assessed using clinical observation for evidence of the characteristic FAS facial anomalies, with formal physical measurement of these features not essential at the screening stage (R2)

18. ... physical measurement of palpebral fissures

19. ... the University of Washington Lip-Philtrum Guide

20. ... the facial photographic screening tool

21. Palpebral fissure length must be assessed using formal physical measurement and comparison with population references at the screening stage

22. Thin upper lip and smooth philtrum must be assessed using formal tools such as the University of Washington Lip-Philtrum Guide at the screening stage

R1-Round 1; R2-Round 2; IQD-inter-quartile deviation.

'Includes responses 'agree' and 'strongly agree'.

Results for statements that reached $70 \%$ agreement (consensus) are presented in bold.

${ }^{1}$ Friedman test indicated a significant difference in agreement with the 3 statements that described different methods to assess growth (statements 11-13: Friedman chi-square $=19.3, p<0.001$ ). Post-hoc testing found a significant difference between ratings for statements 11 and 12 (Wilcoxon $Z=-3.5, p<0.001$ ) and 12 and 13 (Wilcoxon $Z=-3.1, p=0.002$ ).

to have acceptable sensitivity, specificity and costeffectiveness. These findings reflect previously identified difficulties in screening for FASD [20,29,30], and deficits in diagnostic and management capacity to support screening $[9,14,15,29,44,45]$.
We found consensus agreement on the need for a checklist and criteria for referral to support the implementation of screening for FASD. We also found consensus agreement that screening primarily requires health professionals to assess prenatal alcohol exposure 
Table 5 Agreement with statements on screening assessment methods for central nervous system abnormalities in rounds 1 and 2

\begin{tabular}{|c|c|c|c|}
\hline Statement & $\begin{array}{l}\mathrm{R} 1 \\
\mathrm{~N}\end{array}$ & $\begin{array}{l}\text { R1 \% Agree } \\
\text { (IQD) }\end{array}$ & $\begin{array}{l}\text { R2 \% Agree } \\
\text { (IQD) }\end{array}$ \\
\hline \multicolumn{4}{|l|}{ Central nervous system (CNS) abnormalities } \\
\hline \multicolumn{4}{|l|}{ Assessment of CNS abnormalities in FASD screening may include: } \\
\hline 1.... developmental milestones & 80 & $96(1)$ & - \\
\hline 2. ... motor and sensory function & 77 & $88(1)$ & - \\
\hline 3. ... cognition (IQ) & 79 & $92(1)$ & - \\
\hline 4.... memory & 78 & $89(1)$ & - \\
\hline 5. ... academic achievement & 79 & $91(1)$ & - \\
\hline 6.... executive functioning and abstract reasoning & 79 & $89(1)$ & - \\
\hline 7. ... adaptive behaviour & 75 & $92(1)$ & - \\
\hline 8.... attention and hyperactivity & 80 & $95(1)$ & - \\
\hline 9.... communication (receptive and expressive language) & 78 & $94(1)$ & - \\
\hline 10. ... social skills and social communication & 80 & $93(1)$ & - \\
\hline $11 . .$. hard and soft neurologic signs (including sensory-motor signs) & 74 & $87(1)$ & - \\
\hline 12. ... seizures that are not due to a postnatal insult or other postnatal process & 74 & $78(1)$ & - \\
\hline 13.... head circumference & 78 & $96(1)$ & - \\
\hline 14.... brain imaging & 67 & $57(1)$ & - \\
\hline \multicolumn{4}{|l|}{ The choice of tests for neuro-behavioural assessments should be guided by: } \\
\hline $15 . .$. the availability of valid and reliable instruments & 76 & $90(1)$ & - \\
\hline 16.... clinician preference and experience & 74 & $60(2)^{\ddagger}$ & - \\
\hline 17. ... test appropriateness for patient age and cultural background & 78 & $97(1)$ & - \\
\hline 18. At the screening stage it is not necessary to formally assess and measure suspected CNS anomalies & 68 & - & $72(1)$ \\
\hline \multicolumn{4}{|l|}{$\begin{array}{l}\text { At the screening stage the following are acceptable indicators of possible CNS abnormalities (neurological, } \\
\text { functional or structural): }\end{array}$} \\
\hline 19. ... clinical identification & 66 & - & $97(0)$ \\
\hline 20. ... parent or other credible third party report & 66 & - & $91(0)$ \\
\hline $21 . .$. results of previous relevant formal assessments (e.g. psychological report) & 65 & - & $100(1)$ \\
\hline \multicolumn{4}{|l|}{ Birth defects } \\
\hline $\begin{array}{l}\text { 22. FASD screening should assess and record the presence of birth defects as part of the clinical } \\
\text { examination }\end{array}$ & 81 & $99(1)$ & - \\
\hline
\end{tabular}

R1-Round 1; R2-Round 2; IQD-inter-quartile deviation.

'Includes responses 'agree' and 'strongly agree'.

‡Statement excluded from round 2 as proportion agreement was $59.5 \%$.

Results for statements that reached $70 \%$ agreement (consensus) are presented in bold.

and consider it as a possible cause of abnormalities. These findings are consistent with the need for greater awareness of FASD and improved capacity for screening among Australian health professionals [46,47], and highlight support for targeted screening as a strategy which can be integrated into usual clinical practice and passive case finding. Over half of participants agreed that most of the information required for FASD screening is assessed at birth or during relevant clinical presentations in childhood, which indicates some capacity for FASD screening in Australia. However, perceptions of participants in this study could differ systematically from health professionals who do not have experience or expertise in FASD screening or diagnosis.

Participants indicated that standard, explicit and efficient screening criteria are needed to determine when referral for a diagnostic evaluation is required. Few formal, validated instruments for FASD screening exist, and no single instrument is suitable for all ages and settings [29]. The absence of a single common distinguishing feature of FASD that can be used to indicate the need for a diagnostic evaluation has required programs to use aetiological risk factors and diagnostic features to determine whether a diagnostic evaluation is required. 
Although these screening methods have been considered unreliable, time consuming and rudimentary [48], most participants supported the use of screening indicators linked to aetiological and diagnostic factors, and emphasised the need to identify efficient and effective screening criteria.

Inaccurate FASD screening and case ascertainment have been linked to deficits in practitioner training and the use of inconsistent case definitions $[8,26]$. Formal assessment of facial dysmorphology and functional CNS performance at the screening stage was not considered necessary, reliable or appropriate in all settings. The need for valid population reference data for comparison was also identified. Although more than half of participants were not familiar with the formal assessment instruments for alcohol exposure presented, we found consensus agreement on the need for formal assessment methods, and on the usefulness of the AUDIT-C assessment instrument [41] following description of this instrument in round 2 .

The facial photographic screening tool was considered to provide a feasible alternative to the measurement of facial anomalies by inexperienced assessors, although some participants indicated concerns about the use of facial dysmorphology assessment in FASD screening. While facial anomalies have been used to screen for FAS $[26,27,29]$ facial anomalies are not commonly assessed in FASD screening $[9,32]$. The assessment of facial anomalies has been considered inappropriate in general population screening for FASD due to multiple factors, including its low predictive value for FASD [29]. However, diagnostic guidelines and criteria for referral recognise the significance of the characteristic FAS facial anomalies as an indicator of the need for diagnostic evaluation, particularly in the absence of confirmed prenatal alcohol exposure $[6,9,12]$.

Criteria have been established to identify when population screening is appropriate and ethically justified, and to ensure that screening does more good than harm [49]. The variable application of these criteria in practice has been attributed to their inherent subjectivity and a lack of evidence to comprehensively evaluate screening interventions [50]. Criteria for the evaluation of population-based screening interventions include whether the potential impact of the condition is sufficient to justify screening; whether there is a benefit from early diagnosis and treatment; whether there is a cost-effective and acceptable screening test; and whether appropriate diagnostic services and treatment are available [49-51]. Our finding of a lack of consensus on the need for universal screening of apparently healthy individuals is consistent with the failure of FASD screening to meet some of these requirements.

Targeted screening for high risk presentations and groups was supported as a strategy to improve the identification of FASD in Australia, consistent with developments in health policy [23]. Participant comments emphasised the importance of ensuring the effectiveness of screening interventions prior to their use to ensure that the intervention benefits both individuals screened and the community. There is little reliable information on the epidemiology of FASD in Australia, and it is not clear what proportion of individuals with FASD would be identified by targeted screening of high-risk groups. Our findings highlight the complex issues that must be addressed when pursuing the deceptively simple objective of early diagnosis [49], and the real need for systematic evaluation of the risks and benefits of proposed screening interventions [50].

The Delphi method provides a strong basis for the construct validity of the study findings, with participants able to validate their initial responses and identify areas of uncertainty [34]. This study was primarily based on an exploration of established screening methods, and our use of non-representative sampling and a modified Delphi process may have limited our ability to evaluate all potentially relevant information on the design of FASD screening programs in Australia. Nevertheless, participants provided diverse perspectives on the use of screening for FASD, including the identification of risks associated with screening, and the importance of both individual case finding and a population based approach. Further research is needed to evaluate potential strategies to facilitate improved identification of FASD, and to evaluate their performance and acceptability in the Australian context.

We aimed to recruit health professionals who had specific experience or expertise in FASD screening or diagnosis to identify consensus perceptions on the approach to FASD screening in Australia. Paediatricians were most highly represented within the study sample, reflecting the profession's key role in diagnosis in Australia. The round 1 response in this study exceeded the $70 \%$ recommended level [52] and was similar to that observed in other Delphi studies involving clinician panels [53,54]. Attrition was greatest between the time of agreement to participate in the study and returning the round 1 questionnaire, and similar to that reported by others [53]. Delay between recruitment of the panel and distribution of the questionnaire, as well as the recruitment of a large panel [55] may have reduced response in this study. Although we found response completeness was associated with participant occupation and experience, there was little evidence of differences in perceptions about screening according to response completeness.

Evaluation of pilot screening interventions are required to address the lack of evidence to support the effectiveness of screening for FASD in Australia and identify the impact of screening parameters on program outcomes and effectiveness. This approach to development would enable 
investigation of practical barriers to success, including the ability to engage with high risk groups. The development of effective screening interventions can improve our understanding of the epidemiology of FASD and its prevalence in high risk groups; contribute to the identification of appropriate strategies for FASD management and prevention; and provide an empirical evidence base for FASD policy in Australia.

It is clear from our findings that additional strategies are also required to improve passive case-finding, referral and diagnosis in Australia. Signs of FASD are frequently nonspecific, and barriers to seeking or accessing appropriate diagnostic and intervention services are likely to be important obstacles to passive case finding. Strategies that can be used to improve passive case finding capacity and the identification of FASD in Australia include the development of resources and programs to improve awareness of the disorders and their prevention among health professionals, other relevant professionals and the wider community; the development of standard criteria to guide health professionals on appropriate referral of individuals who require specialist assessment, as implemented in North America [6,12]; and improved diagnostic capacity and access to specialist services.

\section{Conclusion}

Our findings provide an agreed basis for the development and evaluation of targeted screening for FASD in high risk groups, standard criteria for referral, and other strategies to improve passive case identification, including improved awareness of the disorders among health professionals. Health professionals require well-defined, effective and ethically justified methods to identify and refer individuals who require specialist diagnostic assessment, and appropriate training to deliver these services. Established capacity for diagnosis and management is also essential, and the development of effective targeted screening interventions can contribute to the identification of appropriate strategies for the design and resourcing of FASD diagnosis, management and prevention services in Australia.

\section{Competing interests}

The authors declare that they have no competing interests.

\section{Authors' contributions}

$C B, E J E$ and JMP designed the study and CB and EJE supervised the study. $C B, E J E, R E W, J L$ and $H J$ designed the study questionnaire, and all authors, including consumer and community representatives, were members of a project steering group that reviewed the study methods and procedures. REW programmed the online questionnaire and analysed the data. REW drafted the manuscript and all authors edited the manuscript. All authors read and approved the final version of the manuscript.

\section{Acknowledgements}

We particularly thank the health professionals who completed the survey. We acknowledge the contributions of Alison Anderson who provided specialist programming advice, Laura Bond who was employed as a project officer, and Dr Bill Kean who was a project observer appointed by the Australian Government Department of Health and Ageing.

This study was funded by the Australian Government Department of Health and Ageing. Individual contributions were also supported by National Health and Medical Research Council (NHMRC) Research Fellowships (CB 634341 and JH 1021252), an NHMRC Program Grant (CB and JMP 572742), NHMRC Practitioner Fellowships (EJE 457084 and 1021480), an NHMRC Enabling Grant (EJE and CB 402784) and an Australian Research Council Future Fellowship (JL FT0991861).

\section{Author details}

${ }^{1}$ Telethon Institute for Child Health Research, Centre for Child Health Research, The University of Western Australia, Perth, Australia. ${ }^{2}$ Discipline of Paediatrics and Child Health, Sydney Medical School, University of Sydney, Sydney, Australia. ${ }^{3}$ The Children's Hospital at Westmead, Sydney, Australia. ${ }^{4}$ The George Institute for Global Health, Sydney, Australia. ${ }^{5}$ Public Health Genetics, Genetic Disorders, Murdoch Childrens Research Institute, Melbourne, Australia. 'Department of Paediatrics, University of Melbourne, Melbourne, Australia. ${ }^{7}$ Centre for Population Health Research, Curtin University, Perth, Australia. ${ }^{8}$ Menzies School of Health Research, Charles Darwin University, Darwin, Australia. ${ }^{9}$ Russell Family Fetal Alcohol Disorders Association, Cairns, Australia. ${ }^{10}$ Centre for Chronic Disease, School of Medicine, University of Queensland, Brisbane, Australia. ${ }^{11} \mathrm{Child}$ and Adolescent Health Service, Department of Health Western Australia, Perth, Australia. ${ }^{12}$ National Organisation for Fetal Alcohol Syndrome and Related Disorders, Adelaide, Australia. ${ }^{13}$ National Drug and Alcohol Research Centre, University of New South Wales, Sydney, Australia. ${ }^{14}$ Nindilingarri Cultural Health Services, Fitzroy Crossing, Australia.

Received: 23 August 2012 Accepted: 21 January 2013

Published: 25 January 2013

\section{References}

1. Bailey BA, Sokol RJ: Prenatal alcohol exposure and miscarriage, stillbirth, preterm delivery, and sudden infant death syndrome. Alcohol Res Health 2011, 34(1):86-91.

2. Jones KL: The effects of alcohol on fetal development. Birth Defects Res $C$ Embryo Today 2011, 93(1):3-11

3. O'Connor MJ, Paley B: Psychiatric conditions associated with prenatal alcohol exposure. Dev Disabil Res Rev 2009, 15(3):225-234.

4. Sokol R, Delaney-Black V, Nordstrom B: Fetal alcohol spectrum disorder. JAMA 2003, 290(22):2996-2999.

5. Astley SJ, Clarren SK: Diagnosing the full spectrum of fetal alcoholexposed individuals: Introducing the 4-Digit Diagnostic Code. Alcohol Alcohol 2000, 35:400-410.

6. Chudley AE, Conry J, Cook JL, Loock C, Rosales T, LeBlanc N: Fetal alcohol spectrum disorder: Canadian guidelines for diagnosis. Can Med Assoc J 2005, 172(5 Suppl):S1-S21.

7. Jones KL, Smith DW, Ulleland CN, Streissguth P: Pattern of malformation in offspring of chronic alcoholic mothers. Lancet 1973, 1(7815):1267-1271.

8. Stratton K, Howe C, Battaglia F: Fetal alcohol syndrome: diagnosis, epidemiology, prevention, and treatment. Institute of medicine. Washington, DC: National Academy Press; 1996.

9. Astley SJ: Profile of the first 1,400 patients receiving diagnostic evaluations for fetal alcohol spectrum disorder at the Washington State Fetal Alcohol Syndrome Diagnostic \& Prevention Network. Can J Clin Pharmacol 2010, 17(1):e132-164.

10. Chudley AE: Fetal alcohol spectrum disorder: counting the invisible mission impossible? Arch Dis Child 2008, 93(9):721-722.

11. Astley SJ: Diagnostic guide for fetal alcohol spectrum disorders: the 4-digit diagnostic code. 3rd edition. Seattle: University of Washington; 2004.

12. Bertrand J, Floyd RL, Weber MK: Guidelines for identifying and referring persons with Fetal Alcohol Syndrome. MMWR 2005, 54(03/02/2005):1-14

13. Hoyme HE, May PA, Kalberg WO, Kodituwakku P, Gossage JP, Trujillo PM, Buckley DG, Miller JH, Aragon AS, Khaole N, et al: A practical clinical approach to diagnosis of Fetal Alcohol Spectrum Disorders: Clarification of the 1996 institute of medicine criteria. Pediatrics 2005, 115(1):39-47.

14. Benz J, Rasmussen C, Andrew G: Diagnosing fetal alcohol spectrum disorder: history, challenges and future directions. Paediatr Child Health 2009, 14(4):231-237 
15. Elliott E, Payne J, Morris A, Haan E, Bower C: Fetal alcohol syndrome: a prospective national surveillance study. Arch Dis Child 2008, 93(9):732-737.

16. Morleo M, Woolfall K, Dedman D, Mukherjee R, Bellis MA, Cook PA: Underreporting of foetal alcohol spectrum disorders: an analysis of hospital episode statistics. BMC Pediatr 2011, 11:14.

17. Bertrand J: Interventions for children with fetal alcohol spectrum disorders (FASDs): overview of findings for five innovative research projects. Res Dev Disabil 2009, 30(5):986-1006.

18. Streissguth AP, Bookstein FL, Barr HM, Sampson PD, O'Malley K, Young JK: Risk factors for adverse life outcomes in fetal alcohol syndrome and fetal alcohol effects. J Dev Behav Pediatr 2004, 25(4):228-238.

19. Clarren SK, Lutke J: Building clinical capacity for fetal alcohol spectrum disorder diagnoses in Western and Northern Canada. Can J Clin Pharmacol 2008, 15(2):e223-237.

20. May PA, Gossage JP, Kalberg WO, Robinson LK, Buckley D, Manning M Hoyme HE: Prevalence and epidemiologic characteristics of FASD from various research methods with an emphasis on recent in-school studies. Dev Disabil Res Rev 2009, 15(3):176-192.

21. Elliott E, Payne J, Haan E, Bower C: Diagnosis of foetal alcohol syndrome and alcohol use in pregnancy: a survey of paediatricians' knowledge, attitudes and practice. J Paediatr Child Health 2006, 42:698-703.

22. Payne J, France K, Henley N, D'Antoine H, Bartu A, O'Leary C, Elliott E, Bower $\mathrm{C}$ : Changes in health professionals' knowledge, attitudes and practice following provision of educational resources about prevention of prenatal alcohol exposure and fetal alcohol spectrum disorder. Paediatr Perinat Epidemiol 2011, 25(4):316-327.

23. Department of Health Western Australia: Fetal alcohol spectrum disorder model of care. Perth. Western Australia: Health Networks Branch, Department of Health; 2010.

24. House of representatives committees, parliament of Australia; http://www.aph. gov.au/Parliamentary_Business/Committees/ House_of_Representatives_Committees?url=spla/fasd/report.htm.

25. Astley SJ, Clarren SK: A fetal alcohol syndrome screening tool. Alcohol Clin Exp Res 1995, 19(6):1565-1571.

26. Weiss M, Cronk CE, Mahkorn S, Glysch R, Zirbel S: The wisconsin fetal alcohol syndrome screening project. Wis Med J 2004, 103(5):53-60.

27. Astley SJ, Stachowiak J, Clarren SK, Clausen C: Application of the fetal alcohol syndrome facial photographic screening tool in a foster care population. J Pediatr 2002, 141:712-717.

28. Mattson SN, Roesch SC, Fagerlund A, Autti-Ramo I, Jones KL, May PA, Adnams CM, Konovalova V, Riley EP: Toward a neurobehavioral profile of fetal alcohol spectrum disorders. Alcohol Clin Exp Res 2010, 34(9):1640-1650.

29. Goh YI, Chudley AE, Clarren SK, Koren G, Orrbine E, Rosales T, Rosenbaum C: Development of Canadian screening tools for fetal alcohol spectrum disorder. Can J Clin Pharmacol 2008, 15(2):e344-366.

30. Mizejewski GJ: Can prenatal screening for fetal alcohol spectrum disorder be justified? a commentary. Gynecol Obstet Invest 2010, 69(2):128-130.

31. Pass KA, Mizejewski GJ: Is it time for newborn screening for fetal alcohol spectrum disorders: a commentary. J Pediatr Sci 2009, 1:e5.

32. Conry J, Asante K: Youth probation officers' guide to FASD screening and referral. British Columbia: The Asante Centre for Fetal Alcohol Syndrome; 2010.

33. Keeney S, Hasson F, McKenna H: The delphi technique in nursing and health research. Chichester: Wiley-Blackwell; 2011.

34. Okoli C, Pawlowski SD: The Delphi method as a research tool: an example, design considerations and applications. Inf Manage 2004, 42:15-29.

35. Elliott L, Coleman K, Suewongpat A, Norris S: Fetal Alcohol Spectrum Disorders (FASD): systematic reviews of prevention, diagnosis and management. Christchurch, New Zealand: Health Services Assessment Collaboration, University of Canterbury; 2008.

36. Watkins RE, Elliott EJ, Mutch RC, Latimer J, Wilkins A, Payne JM, Jones HM, Miers S, Peadon E, McKenzie A, et al: Health professionals' perceptions of the adoption of existing guidelines for the diagnosis of fetal alcohol spectrum disorders in Australia. BMC Pediatr 2012, 12(1):69.

37. Hasson F, Keeney S, McKenna H: Research guidelines for the Delphi survey technique. J Adv Nurs 2000, 32(4):1008-1015.

38. McDonald JH: Handbook of biological statistics. 2nd edition. Baltimore, Maryland: Sparky House Publishing; 2009.

39. Elo S, Kyngas H: The qualitative content analysis process. J Adv Nurs 2008, 62(1):107-115.
40. Streubert-Speziale HJ, Carpenter DR: Qualitative research in nursing: advancing the humanistic imperative. 3rd edition. Philadelphia: Lippincott Williams and Wilkins; 2003

41. Bush K, Kivlahan DR, McDonell MB, Fihn SD, Bradley KA: The AUDIT alcohol consumption questions (AUDIT-C): an effective brief screening test for problem drinking. Ambulatory Care Quality Improvement Project (ACQUIP). Alcohol Use Disorders Identification Test. Arch Intern Med 1998, 158(16):1789-1795.

42. Australian Government Department of Health and Ageing: Pregnancy lifescripts: helping pregnant women prevent alcohol-related harm. Canberra, Australian Capital Territory: Commonwealth of Australia; 2006

43. Russell M: New assessment tools for risk drinking during pregnancy: TACE, TWEAK and others. Alcohol Health Res World 1994, 18(1):55-61.

44. Clarren SK, Lutke J, Sherbuck M: The Canadian guidelines and the interdisciplinary clinical capacity of Canada to diagnose fetal alcohol spectrum disorder. J Popul Ther Clin Pharmacol 2011, 18(3):e494-499.

45. Mutch R, Peadon EM, Elliott EJ, Bower C: Need to establish a national diagnostic capacity for foetal alcohol spectrum disorders. J Paediatr Child Health 2009, 45(3):79-81.

46. Payne J, Elliott E, D'Antoine H, O'Leary C, Mahony A, Haan E, Bower C: Health professionals' knowledge, practice and opinions about Fetal Alcohol Syndrome and alcohol consumption in pregnancy. Aust N Z J Public Health 2005, 29(6):558-564.

47. Payne JM, France KE, Henley N, D'Antoine HA, Bartu AE, Mutch RC, Elliott EJ, Bower C: Paediatricians' knowledge, attitudes and practice following provision of educational resources about prevention of prenatal alcohol exposure and Fetal Alcohol Spectrum Disorder. J Paediatr Child Health 2011, 47(10):704-710

48. Hopkins RB, Paradis J, Roshankar T, Bowen J, Tarride JE, Blackhouse G, Lim M, O'Reilly D, Goeree R, Longo CJ: Universal or targeted screening for fetal alcohol exposure: a cost-effectiveness analysis. J Stud Alcohol Drugs 2008, 69(4):510-519.

49. Wilson JMG, Jungner G: Principles and practice of screening for disease. Public Health Papers No 34. Geneva: World Health Organization; 1968

50. Botkin JR: Assessing the new criteria for newborn screening. Health Matrix Clevel 2009, 19(1):163-186.

51. Wald NJ: The epidemiological approach. 2nd edition. London: Wolfson Institute of Preventive Medicine; 1991

52. Sumsion T: The Delphi technique: an adaptive research tool. $\mathrm{Br} J$ Occup Ther 1998, 61:153-156.

53. Myers $H$, Thomas $E$, Dziedzic K: What are the important components of the clinical assessment of hand problems in older adults in primary care? results of a Delphi study. BMC Musculoskelet Disord 2010, 11:178.

54. Downs J, Bergman A, Carter P, Anderson A, Palmer GM, Roye D, van Bosse $H$, Bebbington $A$, Larsson EL, Smith BG, et al: Guidelines for management of scoliosis in Rett syndrome patients based on expert consensus and clinical evidence. Spine 2009, 34:E607-617.

55. Hsu CC, Sandford BA: The Delphi technique: making sense of consensus. Practical Assessment, Research and Evaluation 2007, 12(10):1-8.

\section{doi:10.1186/1471-2431-13-13}

Cite this article as: Watkins et al:: A modified Delphi study of screening for fetal alcohol spectrum disorders in Australia. BMC Pediatrics 2013 $13: 13$.

\section{Submit your next manuscript to BioMed Central and take full advantage of:}

- Convenient online submission

- Thorough peer review

- No space constraints or color figure charges

- Immediate publication on acceptance

- Inclusion in PubMed, CAS, Scopus and Google Scholar

- Research which is freely available for redistribution 